\section{BOOK FOR THEORISTS}

\section{Value Distribution Theory}

By Leo Sario and Kiyoshi Noshiro. (The University Series in Higher Mathematics.) Pp. xi+236. (Princeton, N.J.: D. Van Nostrand Co., Inc.; London: D. Van Nostrand Co., Ltd., 1966.) 608 .

Complex analysis, like so many other mathematical disciplines, started essentially in the nineteenth century with the pioneering work of Gauss, Riemann, Cauchy and Weierstrass. If $f((z)$ is a regular, that is differentiable, function of a complex variable $z$ in a plane domain $D$ then $\mathrm{f}(z)$ not only possesses derivatives of all orders, but is locally expansible as a Taylor series. From this result many other important and elegant properties can be deduced, which like the above have no analogue for functions of a real variable. Value distribution theory starts with Picard's fundamental theorem, which states that a function $f(z)$ regular in the whole plane, other than a polynomial, assumes all values with at most one excep. tion infinitely often. Meromorphic functions, which are locally ratios of two regular functions, form a natural extension of regular functions. For these $\infty$ is a possible value and two exceptional or Picard values are possible. The theory of meromorphic functions in the open plane on a finite circle was built into an imposing edifice by $R$. Nevanlinna, who, using an idea of Collingwood and Littlewood, significantly extended Picard's result.

In this book the authors consider a further extension, namely maps of one Riemann surface $R$ on to another $S$. Regular functions correspond to the case where $S$ is the open plane, moromorphic functions to that where $S$ is the closed plane including $\infty$. The formidable problems which arise are overcome on the whole very successfully. If $S$ is a compact surface then, following Chern, it is shown that in rather general conditions the largest possible number of Picard values is $-e_{8}$, where $e_{s}$ is the Eulercharacteristic of $S$. I found Chapter 5 particularly interesting, because in it functions meromorphic in the plane outside a perfect set $E$ of essential singularities are discussed. Following Carleson and Matsumoto, conditions on $E$ are given under which such a function can have at most $p+2$ Picard values, where $p$ is any positive integer.

Considering the somewhat abstract nature of the material the exposition is clear. The book will be of interest to function theorists and to differential geometers willing to stray a little beyond the boundaries of their discipline.

\section{W. K. HAYMAN}

\section{MORE SOURCES OF CHEMISTRY}

\section{The International Encyclopedia of Physical Chemistry and Chemical Physics}

Topic 9: Transport Phenomena, Vol. 1. Statistical Mechanical Theories of Transport Processes. By Robert M. Mazo. Pp. xiii + 166. (Oxford, London and New York: Pergamon Press, Ltd., 1967.) 60s. net.

BoltzmanN expressed the transport coefficients of a dilute gas in torms of the non-equilibrium distribution function of molecular velocities. His equation was solved by Chapman and by Enskog early in this century. This story is recounted briefly in the first sixty pages of this book, of which the greater part is an account of efforts made in the past 20 years to develop a rigorous theory that is also valid for donse fluids.

Those efforts fall into four main groups. First there is the "kinetic theory" approach (Enskog, Bogoliubov) in which one secks an expansion in powers of the donsity. Unfortunately, the imperfect methods so far used are plagued with singularitios. A second route (Kirkwood, Rice) is more closely related to the theory of Brownian motion and introduces a rather ill-defined friction. constant. A third (Kubo) is based upon "fluctuationdissipation" relations, whereby a transport coefficient is related to an equilibrium fluctuation. The fourth (Prigogine, Résibois) exploits the resemblance to Schrödinger's equation of Liouville's equation in operator form in order to develop perturbation methods of solution.

It is clear that we lack in this field the rigorous and agreed procedures for relating molecular and macroscopic properties that we have in equilibrium statistical mechanics. The few books so far written are biased towards one or other of the four main routes, but this work is well balanced and, in spite of its brevity, most clear on the nature of the physical assumptions made in each case. Coherence is achieved by relating each approach to Liouville's equation.

Those coming to the subject for the first time may be puzzled by a few omissions. Thus Boltzmann's $H$ theorem is discussed at length, but the relation of $H$ to entropy is not mentioned. One equation is described as "a generalized Fokker-Planck equation", but the equation itself is not defined, although it appears seven times in the index. We are given, however, a brief guide to the technique of functional differentiation, and must pardon some omissions in an author who achieves so much, so clearly, in 166 pages.

J. S. Rowlinson

\section{NIELS BOHR}

Niels Bohr: His Life and Work as Seen by his Friends and Colleagues

Edited by S. Rozental. Pp. $355+60$ photographs. (Amsterdam: North-Holland Publishing Company, 1967.) 29 guilders; $58 s$.

This memorial to Professor Niels Bohr is reverential in tone, which is neither surprising nor reprehensible. For it has long been known that Bohr's great influence on his contemporaries and his students was a magic which worked, in part at least, through his ability to capture the respect and the liking of those who worked with him. He was a friendly quick-witted voluble magnet of a man. One of the virtues of these contributions by Bohr's colleaguesDirac and Heisenberg as well as those now working at the Institute of Theoretical Physics in Copenhagen-is that it includes such a great wealth of anecdote that nobody can fail to appreciate the likability of this great Nordic father-figure. The translation of the first Danish version of this volume is a considerable public service.

The account by Léon Rosenfeld and Erik Rudinger of Bohr's early work in Cambridge and Manchester is particularly valuable for the light it throws on the character of work at the two universities. Bohr spent much of his time at Cambridge in the autumn of 1911 listening to the lectures of Thomson, Larmor and Jeans. According to letters to his brother in Denmark, he was from time to time buoyed up by helpful and encouraging conversations with J. J. Thomson, who at one point promised to read a long thesis which Bohr had written on the properties of electrons in metals-an argument which sought to carry further what Sommerfeld had done to account for metallic characteristics in terms of freely moving electrons. But in the event, Thomson never seemed to find the time for a critical reading of the thesis. Although it scems that Bohr had plenty of other activities with which to occupy himself, it would be asking too much of flesh and blood - even that of a consistently modest man like Bohr - to expect that this disappointment would not have added something to the pleasure with which he moved to Rutherford's laboratory at Manchester in March 1912.

Rosenfeld and Rudinger have drawn on letters by George Hevesy (then at Manchester, later at Copenhagen) to provide a keener understanding of the development of Bohr's thinking at this point at which he had not eontri- 